\title{
Effect of transport phenomena of Cladosporium cladosporioides on decolorization and chemical oxygen demand of distillery spent wash
}

\author{
R. Ravikumar
}

Received: 6 August 2012/Revised: 21 December 2013/Accepted: 11 January 2014/Published online: 4 February 2014 (C) Islamic Azad University (IAU) 2014

\begin{abstract}
The focus of this report is directed towards the influence of transport properties of fermentation broth in degradation and decolorization of distillery spent wash using Cladosporium cladosporioides. Batch experiment was conducted in a bioreactor for 7 days with $250 \mathrm{rpm}$ at $35{ }^{\circ} \mathrm{C}$ under optimum bioprocess conditions maintained using control system. Results of the morphological analysis revealed that $C$. cladosporioides has been observed to form mainly pellets during fermentation under mild agitation. The organism changed from filamentous form to pellet and then breaks, ending with filamentous form. All the models Power law, Bingham plastic, and Herschel-Bulkley fitted the general trend well with high $R^{2}$ values ranging from 0.9 to 0.98 , indicating shear rate thinning nature of the broth. A maximum decolorization of $72.3 \%$ was achieved with chemical oxygen demand reduction of $74.8 \%$ due to the low viscous and pellet nature of the organism, leading to the transport properties of the fluid. Hence, transport phenomena channalyze the metabolic path way easily and leads to a novel approach for the industries to utilize the spent wash with minimum cost.
\end{abstract}

Keywords Distillery spent wash - Decolorization · Morphology $\cdot$ Rheology $\cdot$ Transport phenomena

R. Ravikumar $(\square)$

Department of Biotechnology, Bannari Amman Institute of

Technology, Sathyamangalam, Erode District 638 401,

Tamil Nadu, India

e-mail: ravi_cbe1@rediffmail.com

\section{Introduction}

Ethanol production through bioprocess using molasses yields large volumes of dark brown and highly toxic wastewater and contains considerable amount of organic compounds. Although most of the organic compounds of this type of effluent are removed by means of conventional biodegradation treatments, the dark color due to the presence of melanoidin-type compound remains as the problem (Vahabzadeh et al. 2004). During the fermentation process, fungal morphology is an important parameter which influences the physical properties of fermentation broth. The rheological behavior is closely related to the morphology and biomass concentrations (Blanch and Bhavaraju 1976; Charles 1978; Metz et al. 1979; Tucker and Thomas 1993). The broth rheology determines the transport phenomena in bioreactors and is considered to be the key factor for getting an improved yield of the targeted product (Berovic et al. 1991; Gehrig et al. 1998). Generally two types of growth forms the filamentous and pelleted can be observed during a fungal fermentation. The pelleted form is found to be less viscous than filamentous form (Daniel et al. 1995; Kim et al. 1983). A brief guideline for the selection of the appropriate technologies for specific applications has been evaluated with morphology and rheology factors (Sanghavi et al. 2013a, b; Gupta et al. 2006, 2009, 2010, 2011). It was suggested in the report that morphological parameters measured by image analysis have been used as a good parameter to indicate rheological properties of the culture broth of $C$. acremonium M25 (Lim et al. 2002). During the production of red pigment using $P$. sinclairii characterization of mycelial morphology and broth rheology of fermentation broth in a 5-L bioreactor played an effective role in increasing the production 
(Cho et al. 2002). Reports revealed the effect of morphology with rheology as process parameters for product yield (Gupta et al. 2007a, b; Mittal et al. 2008, 2010; Jaina et al. 2003). However, studies on transport phenomena with morphology and rheology characteristics of fermentation broth on chemical oxygen demand (COD) reduction and decolorization of distillery spent wash have remained unexplored area of research. Hence, this paper was focused to obtain the possible relationship between characteristics of fungal morphology and rheology with biodegradation and decolorization of distillery spent wash using Cladosporium cladosporioides. In the present investigation, the transport phenomena have been correlated with morphology and rheology properties in developing a suitable technology to treat the distillery spent wash. The present study is also a part of our bioprocess and downstream processing laboratory research conducted to scale up to pilot plant level for suitability of industrial acceptance of this novel technology. Overview of challenges in bioprocessing, a thorough knowledge is essential with respect to behavior of the fermentation broth for treating distillery spent wash, which has not been focused so far resulting in new exploration. This research work was carried out in the bioprocess laboratory of biotechnology department on June 5, 2012, at Sathyamangalam, Erode district, Tamil Nadu, India.

\section{Materials and methods}

\section{Distillery spent wash collection}

The molasses spent wash after biomethanation from anaerobic digester was collected aseptically from distillery division of Bannari Amman Distillery Limited, Periyapuliyur, Erode District, Tamilnadu, India. The spent wash was centrifuged at $4,200 \times g$ for $15 \mathrm{~min}$ before use to remove the suspended solids and was stored at $4{ }^{\circ} \mathrm{C}$. The stored anaerobically treated distillery spent wash (ADSW) was then filtered through (Whatman No: 42) filter paper and was diluted with deionized water (Millipore DirectQ.3 UV).

Inoculum preparation and culture conditions

The organism C. cladosporioides was culture collection of our bioprocess laboratory, isolated from distillery spent wash soil disposal site (Ravikumar et al. 2010, 2013a, b). Fungal inoculum was prepared by transferring a loop full of culture from the slant into a 250-mL Erlenmeyer flask, containing $100 \mathrm{~mL}$ potato dextrose broth and was incubated in an orbatory shaker at $250 \mathrm{rpm}$ at $30{ }^{\circ} \mathrm{C}$ at $\mathrm{pH} 4$ for 7 days. Flasks were incubated until the phase of spore formation. Reseeding was carried out every 25 days to maintain the active population. Fully sporulated cultures obtained after 4 days $\left(8 \times 10^{6}\right.$ spores $/ \mathrm{mL}$ counted using haemocytometer) of incubation were for the experiments. In order to avoid the extended growth, the medium was acidified by adding $30 \% \mathrm{HCl}$.

\section{Batch studies}

The experiment was carried out in a glass, mechanically stirred tank in situ fermenter (BioEngineering, Switzerland Model: KLF-2000-W-21339) (0.130 m inner diameter, $0.50 \mathrm{~m}$ height, and $2.5 \mathrm{~L}$ working volume). In order to analyze the $\mathrm{O}_{2} / \mathrm{CO}_{2}$ during the metabolism of the reaction, a gas analyzer (New Brunswick Scientific, USA, EX-2000) was attached. Fermenter was equipped with a double Rushton disk turbine impeller $(0.0854 \mathrm{~m}$ diameter of disk, $0.125 \mathrm{~m}$ impeller blade width, $0.165 \mathrm{~m}$ impeller blade length, $24 \mathrm{~m}$ space between each turbine) and provided with four rectangular baffles (0.01 m width, $0.42 \mathrm{~m}$ height). Air sparger was attached to a distance of $0.2 \mathrm{~m}$ from the bottom. Bioprocess parameters such as $\mathrm{pH}$, temperature, inoculum concentration, and growth factors, i.e., $\%$ carbon and \% nitrogen were maintained as per the results obtained through optimization studies using response surface methodology (RSM) with design of experiment (data not shown). Previously before RSM studies process, parameters and nutrient requirements were optimized using single factorial experiment (Ravikumar et al. 2011). Bioreactor consists of dissolved oxygen probe, $\mathrm{pH}$ probe and it was mounted over a weighing balance. The airflow rate and the temperature were maintained at $1 \mathrm{vvm}$ and $35^{\circ} \mathrm{C}$, respectively. Control system was attached for controlling temperature, $\mathrm{pH}, \mathrm{rpm}$, air flow rate, dissolved oxygen and also measuring biomass weight. Experiment was carried out for 7 days with $250 \mathrm{rpm}$ in a $5 \mathrm{~L}$ (2.5 L working volume), and the samples were withdrawn periodically by creating a back pressure for color and COD analysis after every $4 \mathrm{~h}$. The fermentation medium in the fermenter was inoculated with $2 \%(\mathrm{v} / \mathrm{v})\left(\approx\right.$ of $0.5 \mathrm{~g} \mathrm{~L}^{-1}$ dry equivalent of the cells) of the seed culture. The inoculums age used throughout the fermentation process was 3 days. Consequently samples collected through peristaltic pump 
(Miclins, India) were sent to microbiology laboratory and instrumentation laboratory of our institute for analyzing the morphological and rheological characteristics of fermentation broth. The observance was measured as decrease in optical density of supernatant of treated ADSW medium at $475 \mathrm{~nm}$ on UV spectrophotometer (PerkinElmer model: Lambda 35). $\mathrm{pH}$ was determined by using digital portable $\mathrm{pH}$ meter (Model pt-10P, Sartorius). COD was measured using open reflux method (APHA 1995). Viscosity was measured using Brookfield programmable LVD VII digital viscometer. $\mathrm{CO}_{2}$ was measured using $\mathrm{CO}_{2} / \mathrm{O}_{2}$ gas analyzer (Brunswick Scientific, USA). For inoculum growth, rpm was maintained using orbatory shaker (thermo incubator shaker).

\section{Morphological studies}

Morphological details of the organism during the degradation process were collected and evaluated using an image analyzer (Image Pro 6.3), which is software coupled with an Olympus light microscope attached to a CCD camera. Samples were fixed with an equal volume of fixative $(13 \mathrm{~mL}$ of $40 \%$ formaldehyde, $5 \mathrm{~mL}$ glacial acetic acid with $200 \mathrm{~mL}$ of $50 \%$ ethanol). Each fixed sample $(0.1 \mathrm{~mL})$ was transferred to a slide, air-dried and stained with methylene blue $(0.3$ methylene blue, $30 \mathrm{~mL}$ $95 \%$ ethanol in $100 \mathrm{~mL}$ water) (Packer and Thomas 1990). Morphological factors such as hyphal length, number of arthrospores, roughness factors hyphal thickness, and length of swollen hyphal fragments were measured after sorting and classifying by image analyzing process. Pellet morphology was evaluated using digital camera. Roughness factor of the pellet was calculated using the formula:

Roughness factor $=(\text { pellet perimeter })^{2} /(4 \Pi x$ pellet area $)$

Rheological studies

Rheological properties of fermentation broth measurements were performed from samples collected from the fermenter at regular intervals using Brookfield programmable LVD VII digital viscometer. It is used to determine the shear stress and apparent viscosity at different shear rate. The relationships were interpreted in terms of different rheological models. Transport properties of the fermentation broth such as Newtonian and non-Newtonian behaviors were analyzed from the viscosity data.
Rheological models used to interpret the relationship between shear stress $(\tau)$ and shear rate $(\gamma)$ are as follows:

Herschel-Bulkley model : $\tau=\tau_{\mathrm{o}}+\left(\mathrm{K} \gamma^{-\mathrm{n}}\right)$

Bingham plastic model : $\tau=\tau_{o}+n \gamma$

Power law model : $\tau=K \gamma^{-n}$

where constants $K$ and $n$ represent the flow consistency index and the flow behavior index, respectively. Validation of the model result was evaluated using the regression coefficient value. From the shear stress, the apparent viscosity can be calculated using the following equation:

$\eta=\tau / \gamma$

where $\tau$ is the shear stress $(\mathrm{Pa}), \eta$ is the apparent viscosity (Pa s) and $\gamma$ is the shear rate $\left(\mathrm{s}^{-1}\right)$.

\section{Results and discussion}

\section{Morphological characteristics of Cladosporium cladosporioides}

Figure 1 shows the morphological changes of $C$. cladosporioides during the fermentation of distillery spent wash captured using CCD Camera at (a) tenfold magnification and (b) 100-fold magnification and analyzed using image analyzer software. Morphology of a filamentous microorganism played an important role in metabolic reaction during fermentation process (Metz et al. 1979). Cladosporium cladosporioides was observed to form mainly pellets during submerged culture fermentation under mild agitation with ADSW as a substrate. The pellet morphology is the main parameter in determining the fermentation process output in terms of reducing the characteristics of the spent wash. The pellet morphology of $C$. cladosporioides varied with changes in the COD and the concentration of the melanoidin pigment present in the spent wash as well as during the progress of batch fermentation. Consequently, it was also observed that for the first 2 days, it was hairy in nature and started to form aggregates during 3-4 days. On 5th day, it was fully aggregated, and for remaining days, it slowly breaks, falls to hairy in nature Fig. 2 showed the pellet formation stages of the organism while decolorizing the distillery spent wash. During the 1st day, it was in filamentous form with more stretched hypha, and later on 2nd day, pellet started to form, increased its size and then further growth continued in a regular phase during the next 4 days. However, the pellet strength was much ridged between 4 
Fig. 1 Morphological changes in the mycelia of Cladosporium cladosporioides during the fermentation of distillery spent wash. Images were taken at a tenfold magnification and b 100 -fold magnification. Arabic numerals in the image are the culture period as day
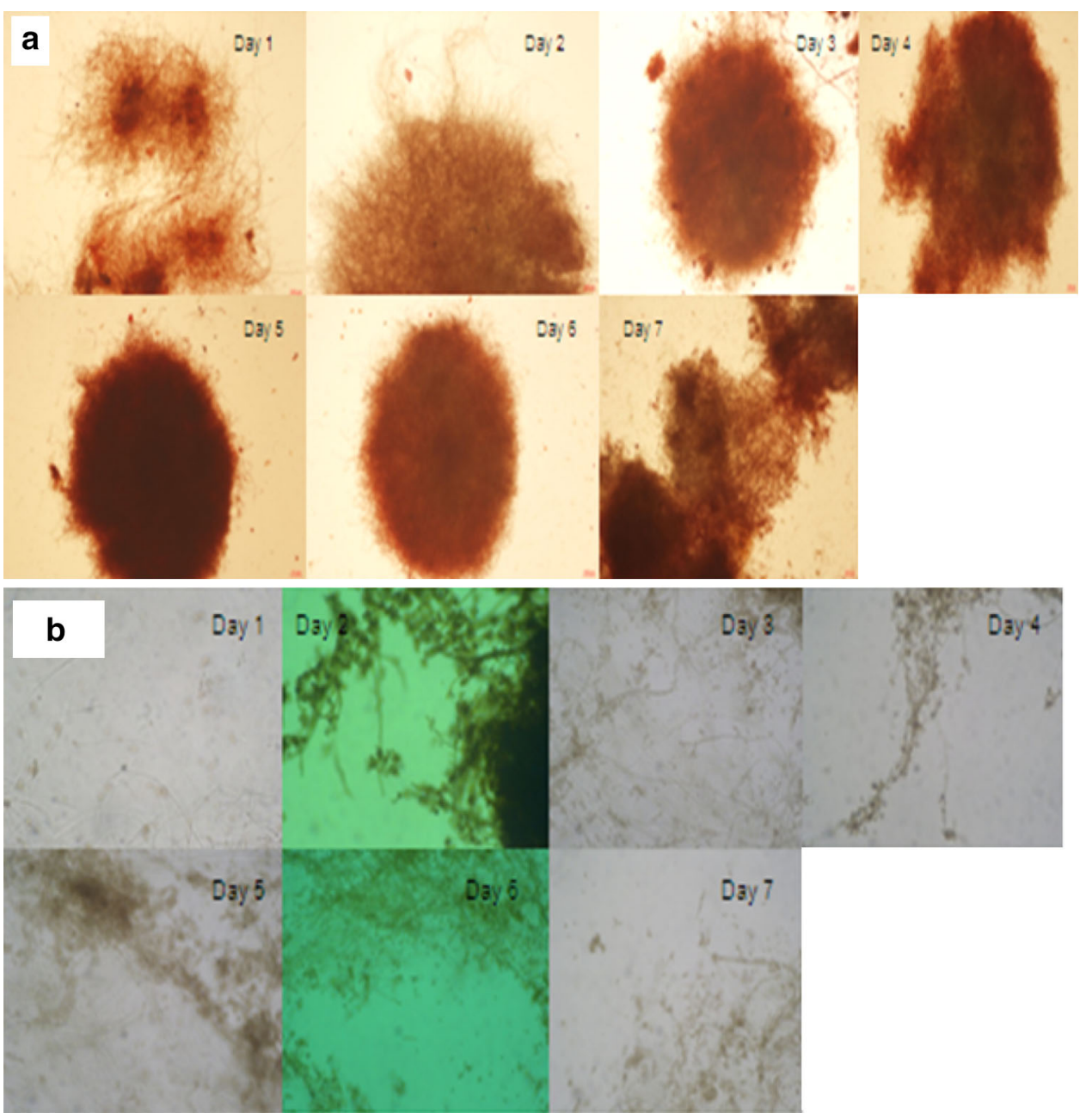

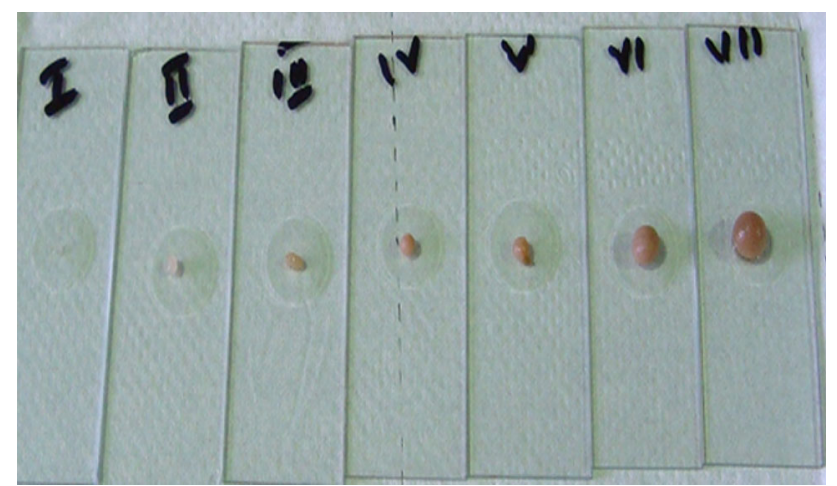

Fig. 2 Photomicrograph of Cladosporium cladosporioides inoculums sample showing pelleted morphology. Roman letters in the image are the culture period as day

and 6 days and later become loosely arranged. This in turn leads to a filamentous mycelia form on the 7 th day. The roughness factor was high during 5-6 days with increase in mean diameter of the pellet. But it was observed that only between 4th and 5th day; the pellet was rigid and firm; and then, it lost its rigidity for the remaining period. It was reported that during the development of A. niger, it lasted for $36 \mathrm{~h}$ to form spherical pellet with short and thick peripheral hyphae (Berovic et al. 1991). In our investigation, pellets formation started at the end of 2 nd day. The change in pellet morphology is due to the phase change from exponential to stationary growth period, and also the biodegrading ability of the fungus was high during this period; Fig. 1 showed the morphological changes of the mycelium sample in the bioreactor. Using image analyzer, it was calculated and found that hyphal length, hyphal thickness, and anthrospores increased till 5th day and then decreased. Morphological changes may have a significant effect on the decolorization of the spent wash. It is also proved to be an influencing factor for the degradation of spent wash in reducing the COD value. But 
even though hyphal length, hyphal thickness and anthrospores increased till 5th day and then decreased, the percentage of decolorization remained constant after 6 days attaining a maximum value along with COD reduction. This is evident from the growth that the organism changed its phase from stationary to death phase. The number of spores formed was high on the 5th day, which is due to the more uptakes of the organic contents of the spent wash resulting in the COD reduction and increase in percentage decolorization.

Reduction in color of the spent wash was also due to the stable nature of the pellet and increase in the hyphal thickness. Similar changes in morphology were reported for biopolymer production using Paecilomyces japonica in batch bioreactor. Rapid pellet formation, breakup of pellet after 7th day, and loss in rigidity were the evidence for the change in morphology (Sinha et al. 2001). The hairness in the pellet increased during the initial stage, aggregated on 5th day and decreased for the remaining 2 days. This was followed by an increase in the roughness, creating an increase in the viscosity of the fermentation broth, which in turn lead to limitations in transport phenomena of the broth. Consequently, the pellet size decreased, and filamentous mycelia were noticed in the broth. In a report, it was mentioned that the exo-biopolymer production that started slowly from the beginning of the fermentation, continually increased even after breakdown of the pellet into fragments (Sinha et al. 2001). In another report, it was mentioned that the cells of $P$. sinclairii formed compact pellets under mild agitation during inoculum preparation but were observed to form filamentous pellet during the entire culture period and also during the exponential phase showed branched longer hyphal length, whereas in our investigation, after the breakdown of the pellet to fragments, the percentage decolorization gradually decreased and remained in constant level. However, the shape of the pellet was observed to change from circular to bulge.

It was decided in a report that the shape of the pellet and agglomeration mainly depend on the aeration and agitation in bioreactor (Smith et al. 1990; Van Suijdam and Metz 1981). Pellet formation, roughness factor, and hyphal measurements were the predominant factors, affecting the decolorization and degradation of the distillery spent wash. Previous reports revealed that the increase in shear stress has an effect on the morphology of G. lucidum, breaking the inoculum pellets into more fragmented hypha and therefore encouraging the development of filamentous form (Buchs and Zoels 2001).
Lee et al. (2004) and Park et al. (1999) revealed that "feather-like" morphology was more favorable than pellets in the production of extracellular polysaccharide and arachidonic acid from Grifola frondosa and Mortierella alpina, respectively. In another report, it was pointed out that more dispersed mycelia observed under controlled dissolved oxygen (DO) conditions were associated with better growth, whereas the predominant clumps present in the uncontrolled DO culture led to more than 2 times higher extracellular polysaccharide productivity (Fazenda et al. 2010). In contrast to the present study, it was observed that degradation and decolorization of the spent wash were high when the pellet formation exists in rigid condition than it was in fragment and mycelia form. It was reported that in general, filamentous growth rather than pellet growth increased the viscosity of the medium (Pazouki and Panda 2000). This is similar to our results but in addition to that the pellet was rigid and stable in nature. It was also observed that extracellular product of enzymeslaccase and cellulase (data not shown) production during the fermentation process with high activity was found when the morphology of the fungus was in pellet form.

\section{Rheological behavior of fermentation broth}

Figure 1 shows the effect of morphological factors of the mycelium sample in the bioreactor during the biodegradation process. Rheological characteristics of a fermentation broth are considered to be an important parameter in describing the transport phenomena of the fermentation process in the reactor. The fermentation broth is a suspension of microorganisms, substrate and organic contents of the distillery spent wash along with melonidine pigment the color causing biopolymer. Earlier reports revealed that an important feature of a batch fermentation process is the change in rheological behavior during fermentation due to the change in substrate composition, biomass concentration, and morphology of the microorganisms (Berovic et al. 1991). It is evident from Fig. 1 that during the decolorization process the viscosity of the fermentation broth increased till 5th day and remained constant, on 6th day, thereafter, it decreased. Viscosity measurement decides the type of fluid and nature of the fermentation broth. It was observed that for first 4 days, the broth behaved as nonNewtonian fluid then slowly changed to Newtonian fluids and remained same even though their was change in pellet morphology. Non-Newtonian behavior is an 
essential feature of fungal fermentation systems, where either the filamentous or the pellet form of mycelia may dominate. Also, at high microbial concentrations, the hyphae can get entangled, resulting in a highly viscous suspension and that may lead to very non-Newtonian behavior. Several studies have shown that fermentation broths containing high concentrations of filamentous microorganisms are highly viscous and are characterized by shear-rate-dependent viscosities and by a yield stress (Ju et al. 1991; Berovic et al. 1993; Goudar et al. 1999). In case of production, the yield is predicted by the measurement of the apparent viscosity of the culture in the production phase, and the morphological characteristics directly determine the rheological properties of the culture broth (Gehrig et al. 1998). The non-Newtonian behavior is relatively predominant at lower shear rate and at higher mycelial concentration. During the stationary phase, the microorganism to deform the three dimensional structure of highly entangled mycelia and to break down the flocs of the mycelial aggregate network, which is negligible at very high shear rates (Kim et al. 1983). The culture broth demonstrated shear rate thinning behavior. This change in viscosity is due to the fact that the pellet roughness increased along with the size and become aggregated with rigidity. Then, the pellet lost its rigidity and disrupted after 5 days resulting in decrease in viscosity. This is also evident from the morphological change. The increase in fluid viscosity makes inconvenient to the process by providing resistance for the transportation of oxygen from the gas film to the core of the cell. There by reducing the biomass growth of the organism. This limitation becomes the rate-limiting step and also a key factor for the decolorization and biomass growth. This resistance factor may be the reason for breaking up of the pellets from 6 to 7 days. This in turn makes the COD reduction constant after attaining maximum percentage of decolorization. The viscous nature of the broth is caused by hydrogen bonding that develops between the carbon source molecules and water in the spent wash. During the interactions between source and water, the hydrogen in the water molecules and the hydrogen in the carbon source molecules have an attraction between each other. Thus, it is the hydrogen bonding that makes the broth viscous. In a report, it was stated that during the production of lovastatin by the filamentous fungus Aspergillus terreus, it was observed that the non-Newtonian rheology of the fermentation broth was influenced both by the biomass concentration and by the size of the fungal pellets (Rodríguez Porcel et al. 2006).

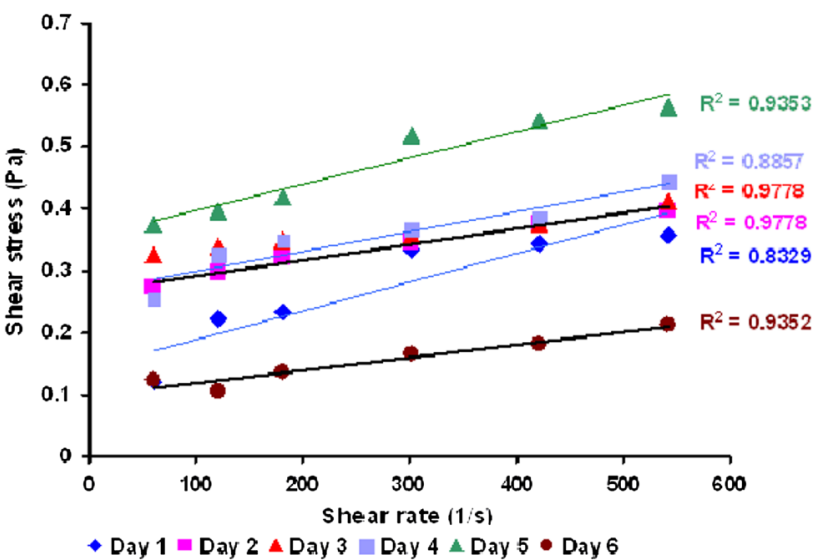

Fig. 3 Power law model for fermentation broth with regression coefficients

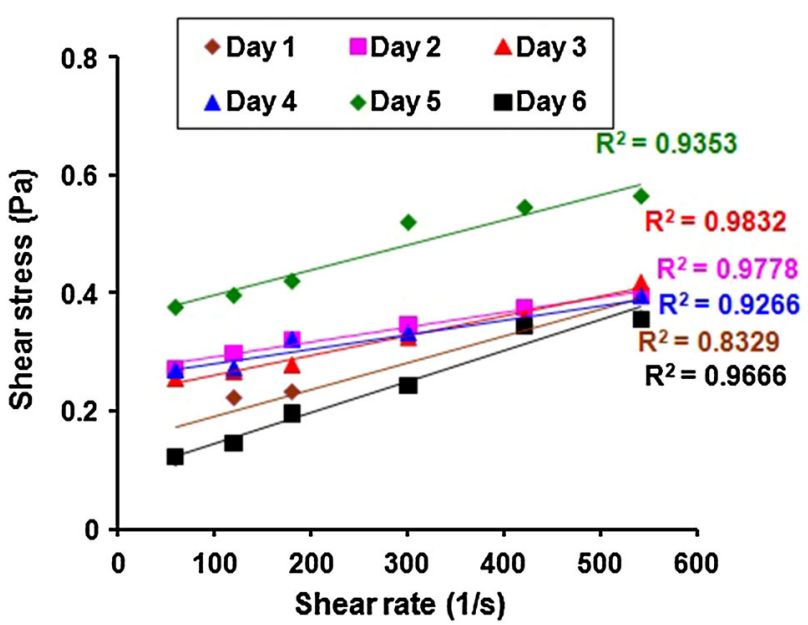

Fig. 4 Bingham plastic model for fermentation broth with regression coefficients

Rheological model applicable to the mycelial broth of Cladosporium cladosporoides

Figures 3, 4, and 5 show shear stress versus shear rate plot made by different rheological models Eqs. 2, 3, and 4 expressing the transport behavior of the fermentation broth of the organism during the decolorization of the distillery spent wash. Figure 4 shows the experimental results of fermentation broth fitted with Power law model. According to the Power law model during the 1 st day, the $R^{2}$ value was 0.832 , which is a lower value because organism was not able to adapt with the available nutrient concentration. The $R^{2}$ value increased in the next level of fermentation due to the increase in 


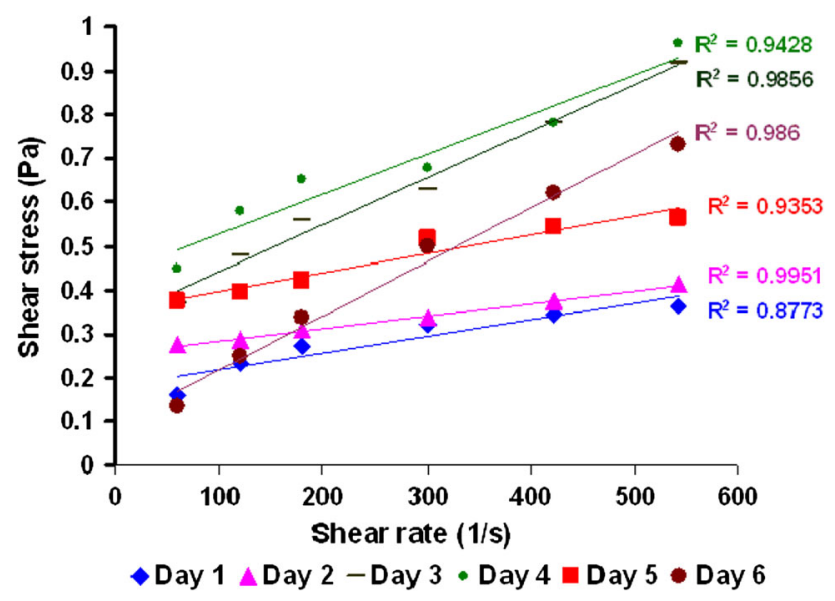

Fig. 5 Herschel-Bulky model for fermentation broth with regression coefficients

biomass concentration. The best fit in the Power law model was observed on 3rd day of fermentation broth $\left(R^{2}\right.$ value $\left.=0.97\right)$ because the organism entered into the exponential phase. These similar results were observed on rheological model applicable to the $C$. acremonium M25 whereas viscosity of the fermentation broth was proportional to the cell concentration (Lim et al. 2002; Cho et al. 2002). From the relationship between viscosity and shear rate for model, it was observed that the viscosity of the fermentation broth increased with an increase in specific growth rate but decreased with increase in shear rate. After $48 \mathrm{~h}$ of fermentation, the broth viscosity was increased along with the formation of pellets because the organism attained the maximum specific growth rate of $0.6897 \mathrm{~h}^{-1}$. This continued up to 5 days after that viscosity of the broth decreased due to the breaking stage of the pellet. These similar results were observed in fermentation broth rheology in exobiopolymer production and also for citric acid production (Sinha et al. 2001). Figure 4 showed the relationship between shear stress and shear rate fitting to Bingham plastic model. In an attempt to find the best fits of the rheological data, various models were selected. All the models fitted the general trend well with high $R^{2}$ values ranging from 0.9 to 0.98 . However, the difference between the fitness of the three models with less than $2 \%$ making the result to interpret. The result implies the presence of yield stress in the broth. It was reported that when Power law model was applied, flow behavior index and consistency coefficient were evaluated; flow behavior index was less than one at all temperatures, confirming the pseudoplastic behavior. Similar trend was reported, implying that it decreased further with the addition of dextran indicating the increase in pseudoplasticity. Consistency coefficient has been found to increase uniformly with the addition of dextran up to $3 \%$. On further addition of dextran, consistency coefficient was found to double (Kaur and Kaler 2008).

Table 1 represents the rheological constants $(K, n)$ obtained through the fitting experimental run data from models. The significant change in the rheological parameters, $K$ and n ranging from 2.336 to 5.476 , from 0.52 to 1.0 , respectively, was observed that $K$ increased rapidly while $\mathrm{n}$ had reached near to a value of 1 . During the fermentation $R^{2}$, value was increased, ranging from 0.8329 to 0.9832 . This model possesses the excellent agreement with the experimental data. This observation was illustrated in Batch cultivation involving the nonNewtonian fluids (Badino et al. 2001). Figure 5 shows the relationship between shear stress and shear rate fitting to Herschel-Bulkey model. The best fit in the Herschel-Bulkey model was observed on 5th day of fermentation broth $\left(R^{2}\right.$ value $\left.=0.995\right)$, and then $R^{2}$ value was decreased due to the disruption of the pellet and hairiness appearance in morphology of the organism. This similar result was observed Rheological model applicable to the C. acremonium M25 (Lim et al. 2002). From the flow behavior index and consistency index through various models, it was confirmed that the fermentation broth behaved as Non-Newtonian pseudoplastic till 5th day and then changed to Newtonian fluid.

Table 1 Rheological constants $(K, n)$ obtained through the fitting experimental run data from models

\begin{tabular}{|c|c|c|c|c|c|c|c|c|c|c|c|c|}
\hline \multirow[t]{2}{*}{ Model equation } & \multicolumn{2}{|l|}{ Day 1} & \multicolumn{2}{|l|}{ Day 2} & \multicolumn{2}{|l|}{ Day 3} & \multicolumn{2}{|l|}{ Day 4} & \multicolumn{2}{|l|}{ Day 5} & \multicolumn{2}{|l|}{ Day 6} \\
\hline & $K(\mathrm{~Pa})$ & $n$ & $K(\mathrm{~Pa})$ & $n$ & $K(\mathrm{~Pa})$ & $n$ & $K(\mathrm{~Pa})$ & $n$ & $K(\mathrm{~Pa})$ & $n$ & $K(\mathrm{~Pa})$ & $n$ \\
\hline Power law & 1.556 & 0.62 & 2.039 & 0.68 & 3.127 & 0.76 & 3.326 & 0.88 & 4.176 & 0.97 & 4.012 & 1.02 \\
\hline Bingham plastic & 2.336 & 0.52 & 2.768 & 0.64 & 3.178 & 0.81 & 4.968 & 0.91 & 5.476 & 0.98 & 5.106 & 1 \\
\hline Hershel bulky & 3.521 & 0.68 & 3.742 & 0.75 & 3.912 & 0.79 & 4.126 & 0.86 & 4.532 & 1 & 4.126 & 1 \\
\hline
\end{tabular}


This is mainly due the biodegradation of the organic matters present in the distillery spent wash with pellet morphological form of the organism.

\section{Bioreactor studies- biodecolorization}

Molasses spent wash is highly recalcitrant waste product because of its melanoidin content, which is a polymer formed by maillard amino-carbonyl reaction. Treatment of distillery spent wash by physical and chemical methods is mainly unsuitable on industrial scale (Raghukumar and Rivonkar 2001). Soil samples were purposefully collected from the disposal sites of distillery unit for screening efficient microorganisms, since chances of getting microbes having the ability to degrade melanoidin are very high in such places. One of such efficient lignocellulosic substance degrading fungi was found to be Cladosporium cladosporioides. Samples were collected at regular interval from the fermenter to obtain the degrading and decolorizing ability of the isolate C. cladosporioides. Part of the sample was sent to rheological study and the broth, to morphological analysis. From Fig. 6, it was observed that during the initial period of 2 days, there is no significant change in the color removal of the spent wash; and maximum decolorization of $72.3 \%$ was achieved with COD reduction of $74.8 \%$ on 5th day. COD reduction and decolorization percentage increased till 5th day and then remained constant. A change in morphology of the organism was noticed from mycelia to pellet adhering to the walls of the impeller and sparger. During the initial phase of growth organism utilities, easily available carbon source was added as medium and later on started to degrade spent wash

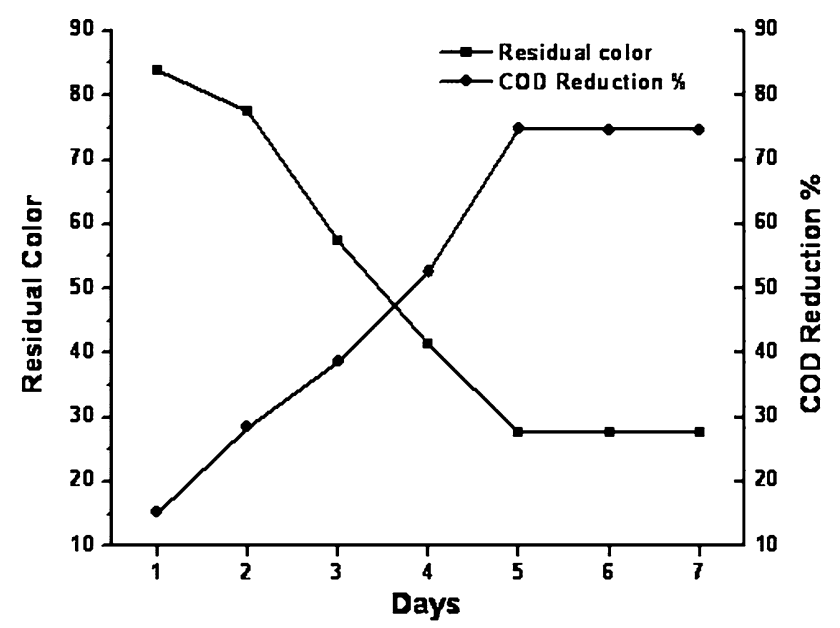

Fig. 6 Time course of degradation and decolorization in the bioreactor with the optimum process conditions components for carbon source. Although spent wash contained large amount of sugar, its easily metabolisable carbon source was almost negligible. So, initially addition of readily available external carbon source like fructose is required for its metabolism. From fig, it was observed that during the initial period of 2 days, there is no significant change in the color removal of the spent wash and maximum decolonization of $72.3 \%$ were achieved with COD reduction of $74.8 \%$ on 5 th day. This is due to the influence of transport phenomena of the fermentation broth. It is evident from the morphological changes and rheological model fitness revealed to the fermentation broth.

\section{Conclusion}

In the present investigation, apart from the conventional approach of controlling the bioprocess parameters required for efficient degradation and decolorization of distillery spent wash, morphological and rheological properties were considered as transport phenomena, which in turn resulted with maximum output and increased the performance of the bioreactor. It is evident from the morphological changes and rheological model fitness with regression coefficient values. The present study clearly demonstrated the ability of C. cladosporioides in biodegradation and decolorization of distillery spent wash, considering transport properties as major factor controlling the overall bioprocess of the bioreactor. Hence, this novel approach reduces the overall cost of the treatment.

Acknowledgments This work is the result of Ph.D. work of the first author. The author wishes to express their gratitude for the support extended by management of Bannari Amman Distillery Division, Periyapuliyur, Erode District, and Tamilnadu, India for providing us the spent wash and also soil sample. We wish to express our thanks to the management of Bannari Amman Institute of Technology, Sathyamangalam, India in carrying out the research in Bioprocess Laboratory, Department of Biotechnology. All the authors are cordially wish to express their thanks to the reviews for their valuable suggestions in improving this research work and manuscript. We also thank Foundation for Revitalization of Local Health Traditions (FRLHT), Bangalore, India for the image analysis and Agharkar Research Institute, Pune, Maharashtra, India (ARI)-National Fungal Culture Collection of India (NFCCI) for DNA sequencing report.

\section{References}

APHA (1995) Standard methods for the examination of waste and wastewater, 15th edn. American Public Health Associations, New York

Badino AC, Facciotti MCR, Schmidell W (2001) Volumetric oxygen transfer coefficients $(\mathrm{kLa})$ in batchcultivations involving nonNewtonian broths. Biochem Eng J 8(2):111-119 
Berovic M, Cimerman A, Steiner W, Koloni T (1991) Submerged citric acid fermentation: rheological properties of Aspergillus niger broth in a stirred tank reactor. Appl Microbiol Biotechnol 34(5):579-581

Berovic M, Koloini T, Olsvik ES, Kristiansen B (1993) Rheological and morphological properties of submerge citric acid fermentation broth in stirred-tank and bubble column reactors. Chem Eng J 53(2):35-40

Blanch HW, Bhavaraju SM (1976) Non-Newtonian fermentation broths: rheology and mass transfer. Biotechnol Bioeng 18(6):745-790

Buchs J, Zoels B (2001) Evaluation of maximum to specific power consumption ratio in shaking bioreactor. J Chem Eng Jpn 34(5):647-653

Charles M (1978) Technical aspects of the rheological properties of microbial cultures. Adv Biochem Eng 8:1-62

Cho YJ, Hwang HJ, Kim SW, Song CH, Yun JW (2002) Effect of carbon source and aeration rate on broth rheology and fungal morphology during red pigment production by Paecilomyces sinclairii in a batch bioreactor. J Biotechnol 95:13-23

Daniel O, Schonholzer F, Zeyer J (1995) Quantification of fungal hyphae in leaves of deciduous trees by automated image analysis. Appl Environ Microbiol 61(11):3910-3918

Fazenda ML, Harvey LM, McNeil B (2010) Effects of dissolved oxygen on fungal morphology and process rheology during fed batch processing of Ganoderma lucidum. J Microbiol Biotechnol 20(4):844-851

Gehrig I, Bart HJ, Anke T, Germerdonk R (1998) Influence of morphology and rheology on the production characteristics of the basidiomycete Cyathus striatus. Biotechnol Bioeng 59(5):525-533

Goudar CT, Strevett KA, Shah SN (1999) Influence of microbial concentration on the rheology of non-Newtonia fermentation broths. Appl Microbiol Biotechnol 51(3):310-315

Gupta VK, Mittal A, Krishnan L, Mittal J (2006) Adsorption treatment and recovery of the hazardous dye, Brilliant Blue FCF, over bottom ash and de-oiled soya. J Colloid Interface Sci 293(1):16-26

Gupta VK, Jain R, Mittal A, Mathur M, Sikarwar S (2007a) Photochemical degradation of the hazardous dye Safranin-T using $\mathrm{TiO}_{2}$ catalyst. J Colloid Interface Sci 309(2):464-469

Gupta VK, Jain R, Varshney S (2007b) Removal of Reactofix goldenyellow 3 RFN from aqueous solution using wheat husk an agricultural waste. J Hazard Mater 142:443-448

Gupta VK, Mittal A, Malviya A, Mittal J (2009) Adsorption of carmoisine A from wastewater using waste materials-Bottom ash and deoiled soya. J Colloid Interface Sci 335(1):24-33

Gupta VK, Rastogi A, Nayak A (2010) Biosorption of nickel onto treated alga (Oedogonium hatei): application of isotherm and kinetic models. J Colloid Interface Sci 342(1):533-539

Gupta VK, Agarwal S, Saleh TA (2011) Chromium removal by combining the magnetic properties of iron oxide with adsorption properties of carbon nanotubes. Water Res 45(6):2207-2212

Jaina K, Gupta VK, Bhatnagar A, Suhas (2003) A comparative study of adsorbents prepared from industrial wastes for removal of dyes. Sep Sci Technol 38(2):463-481

Ju L, Ho CS, Shanahan JF (1991) Effects of carbon dioxide on the rheological behavior and oxygen transfer in submerged penicillin fermentations. Biotechnol Bioeng 38(10):1223-1232

Kaur S, Kaler RSS (2008) Dextran and its effect on the flow behaviour of molasses and crystallization rate. J Food Eng 86:55-60

Kim JH, Lebeault JM, Reuss M (1983) Comparative study on rheological properties of mycelial broth in filamentous and pelleted forms. Eur J Appl Microbiol Biotechnol 18:11-16
Lee BC, Bae JT, Pyo HB, Choe TB, Kim SW, Hwang HJ, Yun JW (2004) Submerged culture conditions for the production of mycelial biomass and exopolysaccharides by the edible Basidiomycete Grifola frondosa. Enzym Microb Technol 35(5):369-376

Lim JS, Kim JH, Kim C, Kim SW (2002) Morphological and rheological properties of culture broth of Cephalosporium acremonium M25. Korea-Aust Rheol J 14:11-116

Metz B, Kossen F, Van Suijdam JC (1979) The rheology of mold suspensions. Adv Biochem Eng 11:104-156

Mittal A, Gupta VK, Malviya A, Mittal J (2008) Process development for the batch and bulk removal and recovery of a hazardous, water-soluble azo dye (Metanil Yellow) by adsorption over waste materials (Bottom Ash and De-Oiled Soya). J Hazard Mater 151(2-3):821-832

Mittal A, Mittal J, Malviya A, Kaur D, Gupta VK (2010) Adsorption of hazardous dye crystal violet from wastewater by waste materials. J Colloid Interface Sci 343(2):463-473

Packer HL, Thomas CR (1990) Morphological measurement on filamentous micro organisms by fully automatic image analysis. Biotechnol Bioeng 35:870-881

Park EY, Koike Y, Higashiyama K, Fujikawa S, Okabe M (1999) Effect of nitrogen source on mycelial morphology and arachidonic acid production in cultures of Mortierella alpina. J Biosci Bioeng 88:61-67

Pazouki M, Panda T (2000) Understanding the morphology of fungi. Bioprocess Eng 22:127-143

Raghukumar C, Rivonkar G (2001) Decolorization of molasses spent wash by white-rot fungus Flavodon flavus, isolated from a marine habitat. Appl Microbiol Biotechnol 55:510-514

Ravikumar R, Monash P, Derek Chan JC, Saravanan K (2010) Microbial decolorization of biomethanate distillery spentwash using Aspergillus nidulans. Asian J Microbiol Biotech Enviro Sci 12:337-342

Ravikumar R, Vasanthi NS, Saravanan K (2011) Single factorial experimental design for decolorizing anaerobically treated distillery spent wash using Cladosporium cladosporioides. Int J Environ Sci Tech 8:97-106

Ravikumar R, Vasanthi NS, Saravanan K (2013a) Biodegradation and decolorization of distillery spent wash with product release by a novel strain Cladosporium cladosporioides: optimization and biokinetics. Chem Biochem J Q 27(3):373-383

Ravikumar R, Renuka K, Sindhu V, Malarmathi KB (2013b) Response surface methodology and artificial neural network for modeling and optimization of distillery spent wash treatment using Phormidium valderianum BDU 140441. Pol J Env Stud 22(4):20-28

Rodríguez Porcel EM, Casas López JL, Sánchez Pérez JA, Fernández Sevilla JM, García Sánchez JL, Chisti Y (2006) Aspergillus terreus broth rheology, oxygen transfer, and lovastatin production in a gas-agitated slurry reactor. Ind Eng Chem Res 45:4837-4843

Sanghavi BJ, Mobina SM, Mathurb P, Lahirib GK, Srivastavaa AK (2013a) Biomimetic sensor for certain catecholamines employing copper(II) complex and silver nanoparticle modified glassy carbon paste electrode. Biosens Bioelectron 39(2):124-132

Sanghavi BJ, Sitaula S, Griep MH, Karna SP, Ali MF, Swami NS (2013b) Real-time electrochemical monitoring of adenosine triphosphate in the picomolar to micromolar range using graphene-modified electrodes. Anal Chem 85(17):8158-8165

Sinha J, Bae JT, Park JP, Song CH, Yun JW (2001) Effect of substrate concentration on broth rheology and fungal morphology during exo-biopolymer production by Paecilomyces japonica in a batch bioreactor. Enzym Microb Technol 29(6):392-399 
Smith JJ, Lilly MD, Fox RI (1990) The effect of agitation on the morphology and penicillin production of Penicillium chrysogenum. Biotechnol Bioeng 35:1011-1023

Tucker KG, Thomas CR (1993) Effect of biomass concentration and morphology on the rheological parameters of Penicillium chrysogenum fermentation broths. Trans Chem Eng 71:111-117

Vahabzadeh F, Mehranian M, Saatari AR (2004) Color removal ability of Phanerochaete chrysosporium in relation to lignin peroxides and manganese peroxides produced in molasses wastewater. World J Microbiol Biotechnol 20:859-864

Van Suijdam JC, Metz B (1981) Influence of engineering variables upon themorphology of filamentous molds. Biotechnol Bioeng 23:111-148 